\title{
Corrosión electroquímica de medios de molienda y efecto de aniones presentes en aguas industriales ${ }^{(\cdot)}$
}

\author{
Luis Magne*, Patricio Navarro*, Cristian Vargas* y Sergio Carrasco*
}

Resumen

El acero utilizado como medios de molienda (bolas o barras) en el procesamiento de minerales, es una materia prima importante desde el punto de vista de costo del proceso. Considerando la importancia del consumo de acero en estos procesos, este trabajo está orientado a evaluar, a escala de laboratorio, el efecto de los aniones presentes en las aguas industriales sobre la corrosión electroquímica de medios de molienda. Para ello se realizaron ensayos en celda electroquímica, midiendo potenciales y corrientes de corrosión a cuatro electrodos que se fabricaron utilizando muestras suficientemente puras de calcopirita, bornita, enargita y.un trozo de bola de acero. Los iones utilizados en los ensayos fueron cloruros, sulfatos, nitratos y carbonatos en concentraciones desde 1 a $180 \mathrm{ppm}$, en forma individual o en mezclas de ellos, de acuerdo a la medición de niveles de éstos en aguas industriales de proceso.

Palabras clave Corrosión. Medios de molienda. Aniones. Aguas industriales.

\section{Electrochemical corrosion of grinding media and effect of anions present in industrial waters}

\begin{abstract}
The steel used in the minerals processing as grinding media (balls or bars), is an important input in terms of cost of the process. Considering the importance of the steel consumption in these processes, this work is guided to evaluate to laboratory scale the effect of the anions present in the industrial waters on the electrochemical corrosion of grinding media. Tests in electrochemical cell, were accomplished measuring potential and corrosion current to four electrodes that were manufactured using sufficiently pure samples of chalcopirite, bornite, enargite and steel ball. The ions used in the tests were chlorides, sulfates, nitrates and carbonates in concentrations from 1 to $180 \mathrm{ppm}$ in individual form or in mixtures, according to the levels measurement of these in industrial waters.
\end{abstract}

Keywords Corrosion. Grinding media. Anions. Industrial waters.

\section{INTRODUCCIÓN}

El consumo de medios de molienda representa una parte importante en los costos de operación de una planta de procesamiento de minerales. A nivel nacional, considerando la gran y mediana minería, el consumo de acero como medio de molienda es del orden de $73.000 \mathrm{t}$ anuales y representa un costo aproximado de 40 millones de dólares ${ }^{[1]}$.

En la molienda húmeda de minerales el consumo de acero aumenta drásticamente, debido principalmente a la componente corrosiva del medio $^{[2 \text { y } 3]}$. Muchos investigadores coinciden en que el desgaste total es ocasionado por un efecto sinergético de corrosión y solicitaciones mecánicas
(Corrosión-Impacto-Abrasión). Sin embargo, es difícil separar la contribución relativa de cada mecanismo en el consumo global ${ }^{[4-6]}$.

Desde el punto de vista electroquímico, en las pulpas de molienda existe una serie de minerales electroactivos que poseen un potencial de reposo más noble que el de los aceros de los medios de molienda. Esto puede dar origen a un ataque de tipo electroquímico al establecerse pilas galvánicas, especialmente en presencia de oxígeno ${ }^{[7-9]}$. Las curvas de polarización típicas de las reacciones principales se muestran en la figura 1 . La reacción catódica, principalmente, es la reducción de oxígeno. Es posible que ocurran otras reacciones de menor importancia, como la reducción de cationes

(•) Trabajo recibido el día 22 de octubre de 1999 y aceptado en su forma final el día 13 de octubre de 2000.

$\left({ }^{*}\right)$ Departamento de Ingeniería Metalúrgica, Universidad de Santiago de Chile, Avda. L.B. O’Higgins 3363, Casilla 10233, Santiago Chile (Chile). E-mail:Imagne@lauca.usach.cl; pnavarro@lauca.usach.cl; cvargas@lauca.usach.cl 


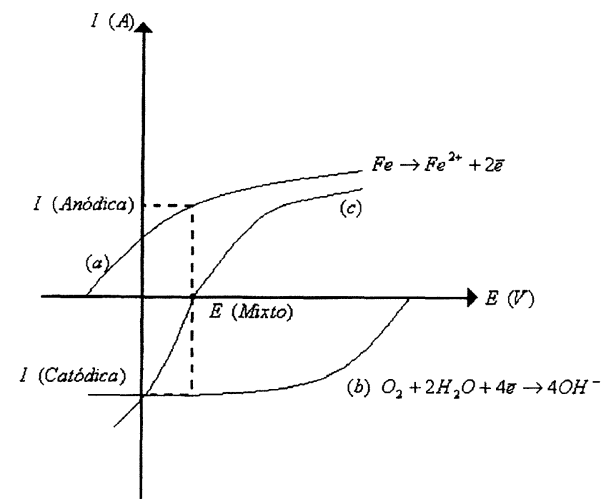

Figura 1. Curvas de polarización anódica del hierro y catódica de reducción del oxígeno.

Figure 1. Anodic and cathodic polarization curves for the iron and oxygen respectively.

reducibles al potencial mixto de corrosión. Según las curvas de polarización catódica de reducción de oxígeno, cuando aumenta la concentración de oxígeno o se agita la solución, aumenta la corriente de oxidación, significando un aumento de la velocidad de corrosión. El burbujeo de un gas inerte disminuye la concentración de oxígeno, por lo tanto la corriente de corrosión disminuye. En el caso límite en que la concentración de oxígeno tiende a cero, el potencial mixto, $E_{\mathrm{m}}$, tiende hacia el potencial de equilibrio termodinámico, $E_{\mathrm{th}}$, del sistema $\mathrm{Fe} / \mathrm{Fe}^{+2}$.

Un modelo de corrosión electroquímica para bolas de molienda, se muestra en la figura 2. Este modelo considera que existe una celda de abrasión diferencial (a) y una celda galvánica mineral-bola (b). En la celda (a), las áreas erosionadas en una bola pueden servir como ánodos promoviendo el consumo de acero, con reducción de oxígeno en el área no erosionada o en el film de óxido pasivante. Con la presencia de un mineral electroactivo, tal como calcopirita y magnetita, las bolas de molienda y el mineral electroactivo forman pares galvánicos, como se muestran en el caso (b), con la superficie de la bola sirviendo como ánodo y la partícula de mineral en contacto actuando como cátodo, donde toma lugar la reducción de oxígeno ${ }^{[10]}$.

Gran parte de los estudios anteriormente citados se realizaron utilizando como base alguna concentración estándar de cloruro o algún otro ion o, en su defecto, agua potable. Dichos estudios no consideran que las aguas de proceso industrial constituyen un medio con características muy diferentes al de soluciones estándar y al agua potable, conteniendo cantidades apreciables de otros iones

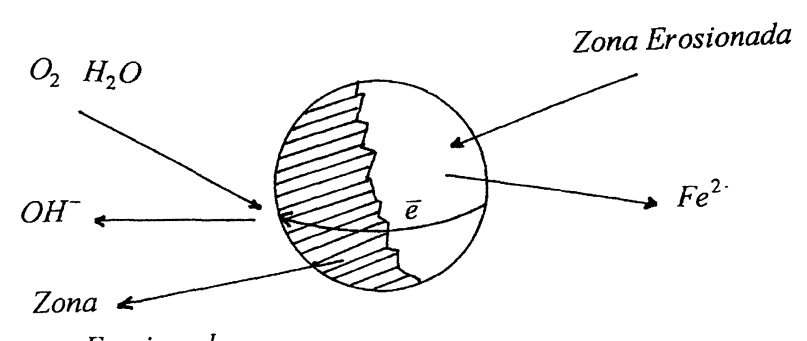

no Erosionada

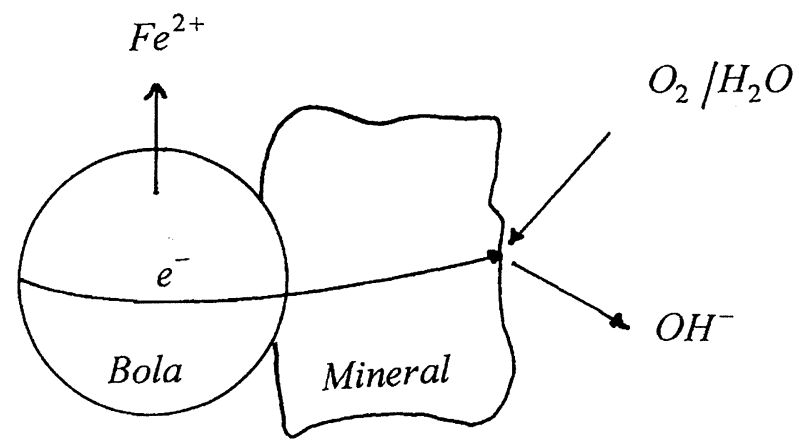

Figura 2. Modelo de corrosión electroquímica de medios de molienda.

Figure 2. Electrochemical corrosion model of grinding media.

como carbonatos, nitratos, sulfatos de hierro y cobre entre muchos otros.

De esta forma el presente estudio electroquímico sobre la corrosión de medios de molienda, considera la presencia de iones como los mencionados anteriormente, evaluando tanto su efecto individual como el efecto de mezclas de ellos. Por ello se pretende evaluar el efecto de aniones presentes en aguas industriales de plantas de molienda de minerales, sobre la corrosión electroquímica de medios de molienda, considerando cloruros, sulfatos, carbonatos y nitratos, como los iones más representativos de estas aguas.

\section{PARTE EXPERIMENTAL}

Las soluciones o electrólitos utilizados para el desarrollo experimental se prepararon en base a agua destilada estándar, con una conductividad eléctrica de $22 \mu \mathrm{S} / \mathrm{cm}$, a la que se agregó la cantidad adecuada de sales para obtener la concentración deseada del ion o iones a evaluar.

En la tabla I, se muestra la caracterización química de agua industrial típica utilizada como base para los ensayos de corrosión (corresponde a agua de alimentación y descarga de un molino de bolas industrial en el que se procesa mineral de cobre sulfurado). Se puede apreciar que los iones con mayor concentración son los cloruros y los carbonatos, 
Tabla I. Caracterización química del agua industrial usada.

Table I. Chemical characterization of industrial water used.

\begin{tabular}{lcc}
\hline & $\begin{array}{c}\text { Agua fresca } \\
\text { de planta } \\
\text { mg/l }\end{array}$ & $\begin{array}{c}\text { Agua descarga } \\
\text { molino } \\
\text { mg/l }\end{array}$ \\
\hline Carbonatos & 40,4 & 73,4 \\
Cloruro & 29,5 & 87,0 \\
Cobre & 0,10 & 0,25 \\
Hierro (II) & $<0,01$ & $<0,01$ \\
Hierro Total & $<0,01$ & $<0,01$ \\
Nitrógeno Total & 7,8 & 23,3 \\
Conductividad, $\mathrm{mS} / \mathrm{cm}$ & 2,3 & 4,2 \\
$\mathrm{pH}$ & 11,30 & 11,50 \\
\hline
\end{tabular}

siguiendo la presencia de iones de hierro y cobre. Finalmente se encuentra el nitrógeno, que puede estar disuelto como $\mathrm{N}_{2}$ o como nitrato. Como se puede apreciar también en esta tabla, la concentración de iones agresivos como el cloruro aumenta drásticamente a la salida del molino hasta valores cercanos al doble del que se tiene a la entrada, lo cual indica la alta disolución de especies minerales presentes en la mena mineral.

De acuerdo a los resultados del análisis de agua industrial, mostrados en la tabla I, se decidió trabajar con las concentraciones de iones que se indican en la tabla II.

Para la preparación de las soluciones se utilizaron los siguientes compuestos químicos sólidos, grado analítico: cloruro de sodio, sulfato de hierro, sulfato de cobre, nitrato de sodio y carbonato de sodio.

Para cumplir con los objetivos planteados, se desarrollaron dos tipos de experiencias, que a continuación se detallan:

- Mediciones de potencial mixto a los electrodos minerales (calcopirita, $\mathrm{CuFeS}_{2}$, bornita, $\mathrm{Cu}_{5} \mathrm{FeS}_{4}$, y enargita, $\mathrm{Cu}_{3} \mathrm{AsS}_{4}$ ) y al medio de molienda, en soluciones que contienen un ion o mezclas de ellos. En esta etapa,se determinaron las concentraciones de iones que producen las mayores diferencias de potencial $(\Delta E)$ entre bola y mineral.

- Obtención del potencial mixto y corriente de corrosión para el sistema bola-oxígeno, utilizando diagramas de Evans para las concentraciones de iones que presentaron la mayor diferencia de potencial entre bola y mineral en el punto anterior.
Tabla II. Concentración de iones utilizados para los ensayos de corrosión.

Table II. Ion concentrations used for the corrosion tests.

\begin{tabular}{|c|c|}
\hline Ion en estudio & $\begin{array}{c}\text { Concentraciones a estudiar } \\
\mathrm{mg} / \mathrm{l}\end{array}$ \\
\hline \multirow[t]{5}{*}{ Cloruro } & $20\left(^{*}\right)$ \\
\hline & 40 \\
\hline & $90\left(^{* *}\right)$ \\
\hline & 150 \\
\hline & $180\left({ }^{* * *}\right)$ \\
\hline \multirow[t]{4}{*}{ Sulfato } & $1\left(^{*}\right)$ \\
\hline & 3 \\
\hline & $5(* *)$ \\
\hline & $10\left({ }^{* * *}\right)$ \\
\hline \multirow[t]{5}{*}{ Carbonato } & $10\left(^{*}\right)$ \\
\hline & 20 \\
\hline & $40\left(^{* *}\right)$ \\
\hline & 80 \\
\hline & $150(* * *)$ \\
\hline \multirow[t]{5}{*}{ Nitratos } & $3(*)$ \\
\hline & 5 \\
\hline & $\left.10{ }^{(* *}\right)$ \\
\hline & 25 \\
\hline & $50(* * *)$ \\
\hline \multirow[t]{3}{*}{ Mezclas de iones } & $\begin{array}{l}\text { Mezcla Baja (contiene las } \\
\text { concentraciones }{ }^{(*)} \text { ). }\end{array}$ \\
\hline & $\begin{array}{l}\text { Mezcla Media (contiene las } \\
\left.\text { concentraciones }{ }^{(* *)}\right) \text {. }\end{array}$ \\
\hline & $\begin{array}{l}\text { Mezcla Alta (contienen las } \\
\text { concentraciones }{ }^{(* * *)} \text {. }\end{array}$ \\
\hline
\end{tabular}

\subsection{Preparación de electrodos de trabajo y celda electroquímica}

Los electrodos de trabajo se obtuvieron de una bola de molienda de acero forjado y los electrodos de mineral a partir de especies naturales suficientemente puras de calcopirita, bornita y enargita.

Los electrodos de trabajo (minerales y acero) fueron montados en resinas epóxicas. Una vez que solidificó la resina, se hizo un orificio de $2 \mathrm{~mm}$ de diámetro para introducir un cable de cobre aislado. El contacto eléctrico entre las probetas y el cable conductór se efectuó mediante una gota de mercurio, sellándose con una mezcla no conductora de la electricidad. Luego se procedió a descubrir la cara de los electrodos que serian empleados para realizar las experiencias. Para esto se desbastó la resina mediante piedra esmeril y posteriormente se pulieron en papel abrasivo, para terminar con un pulido fino en paño giratorio con solución de alúmina de $0,6 \mu \mathrm{m}$ en agua destilada. Este procedimiento se repitió para cada solución en estudio. Los ensayos se realizaron en un reactor de vidrio, de $500 \mathrm{ml}$ de 
capacidad, donde se introdujeron los electrodos de trabajo y referencia, junto con el electrodo auxiliar. Como electrodo de referencia se utilizó el electrodo de calomelano saturado, $245 \mathrm{mV} / \mathrm{ENH}$. La agitación del sistema se realizó con un agitador magnético.

\section{RESULTADOS Y DISCUSION}

\subsection{Potenciales mixtos de los electrodos en diferentes ambientes}

Los resultados de potencial mixto de los electrodos en estudio para diferentes concentraciones de iones se muestran en la tabla III. Se puede observar, que el potencial mixto del medio de molienda presenta valores siempre negativos, independiente del tipo de ion utilizado, en el rango de -300 a -450 $\mathrm{mV} / \mathrm{ECS}$. La bornita, en cambio, presenta potenciales levemente inferiores a cero, del orden de -10 a $-30 \mathrm{mV} / \mathrm{ECS}$. Los potenciales mixtos de la enargita son positivos, del orden de 10 a $40 \mathrm{mV} / \mathrm{ECS}$.

Tabla III. Potenciales mixtos de los electrodos en diferentes ambientes

Table III. Mixed potentials for different conditions

\begin{tabular}{|c|c|c|c|c|c|}
\hline Ion & $\begin{array}{l}\text { Concen- } \\
\text { tración } \\
\text { ppm }\end{array}$ & $\begin{array}{c}\text { Bola } \\
\mathrm{mV} / \mathrm{ECS}\end{array}$ & $\begin{array}{l}\text { Bornita } \\
\mathrm{mV} / \mathrm{ECS}\end{array}$ & $\begin{array}{l}\text { Enargita } \\
\mathrm{mV} / \mathrm{ECS}\end{array}$ & $\begin{array}{c}\text { Calcopirita } \\
\mathrm{mV} / \mathrm{ECS}\end{array}$ \\
\hline \multirow[t]{5}{*}{ Cloruro } & 20 & -308 & $-7,5$ & 39,5 & 285 \\
\hline & 40 & -358 & $-20,0$ & 26,9 & 276 \\
\hline & 90 & -368 & $-25,5$ & 26,7 & 273 \\
\hline & 150 & -382 & $-20,5$ & 29,7 & 266 \\
\hline & 180 & -377 & $-18,5$ & 25,0 & 263 \\
\hline \multirow[t]{4}{*}{ Sulfato } & 1 & -295 & $-9,2$ & 44,0 & 251 \\
\hline & 3 & -333 & $-22,0$ & 14,5 & 242 \\
\hline & 5 & -363 & $-29,0$ & 14,5 & 204 \\
\hline & 10 & -367 & $-30,5$ & 10,9 & 193 \\
\hline \multirow[t]{5}{*}{ Nitrato } & 3 & -290 & $-30,0$ & 22,3 & 249 \\
\hline & 5 & -311 & $-26,9$ & 14,0 & 245 \\
\hline & 10 & -295 & $-25,8$ & 15,0 & 240 \\
\hline & 25 & -310 & $-27,2$ & 14,5 & 235 \\
\hline & 50 & -345 & $-25,4$ & 15,0 & 225 \\
\hline \multirow[t]{5}{*}{ Carbonato } & 10 & -363 & $-14,3$ & 32,9 & 251 \\
\hline & 20 & -338 & $-23,1$ & 20,6 & 214 \\
\hline & 40 & -361 & $-28,9$ & 14,0 & 195 \\
\hline & 80 & -395 & $-21,1$ & 21,0 & 213 \\
\hline & 150 & -428 & $-22,5$ & 18,0 & 199 \\
\hline \multicolumn{2}{|l|}{ Mezcla baja } & -354 & $-18,7$ & 27,5 & 249 \\
\hline \multicolumn{2}{|c|}{ Mezcla media } & -377 & $-23,0$ & 29,0 & 184 \\
\hline \multicolumn{2}{|l|}{ Mezcla alta } & -455 & $-21,5$ & 24,9 & 200 \\
\hline
\end{tabular}

Finalmente la calcopirita presenta potenciales mixtos positivos y muy altos, comprendidos entre 200 y $280 \mathrm{mV} / \mathrm{ECS}$.

En la tabla IV se muestran las diferencias de potencial del par bola-mineral. Se puede apreciar que al aumentar la concentración de cualquier ion en estudio, en general, aumenta también la diferencia de potencial del par bola-mineral.

Para el caso del par bola-bornita se observa que, con una concentración de 150 ppm de ion cloruro se alcanza la máxima diferencia de potencial, siendo ésta de $361,5 \mathrm{mV}$ y para una concentración mayor es, prácticamente, el mismo. Al utilizar sulfato, se alcanza una diferencia de potencial máximo de $334 \mathrm{mV}$, con una concentración del ion de $5 \mathrm{ppm}$. Con $50 \mathrm{ppm}$ de nitrato se obtiene $319,6 \mathrm{mV}$ y con $150 \mathrm{ppm}$ de carbonato $405,5 \mathrm{mV}$. $\mathrm{Al}$ utilizar una mezcla alta de iones, se obtiene una diferencia de potencial máximo de 433,5 mV.

En el sistema bola-enargita, se obtienen diferencias de potencial levemente superiores a las

Tabla IV. Diferencia de potencial bola - mineral en diferentes ambientes.

Table IV. Ball - mineral potential difference in different conditions.

\begin{tabular}{|c|c|c|c|c|}
\hline Ion & $\begin{array}{l}\text { Concen- } \\
\text { tración } \\
\text { ppm }\end{array}$ & $\begin{array}{c}\text { Bola- } \\
\text { bornita } \\
\mathrm{mV}\end{array}$ & $\begin{array}{c}\text { Bola- } \\
\text { enargita } \\
\mathrm{mV}\end{array}$ & $\begin{array}{c}\text { Bola- } \\
\text { calcopirita } \\
\mathrm{mV}\end{array}$ \\
\hline \multirow[t]{5}{*}{ Cloruro } & 20 & 300,5 & 347,6 & 593,0 \\
\hline & 40 & 338,0 & 384,9 & 634,0 \\
\hline & 90 & 343,0 & 394,7 & 641,0 \\
\hline & 150 & 361,5 & 411,7 & 648,0 \\
\hline & 180 & 358,5 & 410,2 & 640,0 \\
\hline \multirow[t]{4}{*}{ Sulfato } & 1 & 285,8 & 339,0 & 546,0 \\
\hline & 3 & 311,0 & 347,5 & 575,0 \\
\hline & 5 & 334,0 & 377,5 & 567,0 \\
\hline & 10 & 333,5 & 377,9 & 560,0 \\
\hline \multirow[t]{5}{*}{ Nitrato } & 3 & 260,0 & 312,3 & 539,0 \\
\hline & 5 & 284,1 & 325,0 & 556,0 \\
\hline & 10 & 269,2 & 310,0 & 535,0 \\
\hline & 25 & 282,8 & 324,5 & 545,0 \\
\hline & 50 & 319,6 & 360,0 & 570,0 \\
\hline \multirow[t]{5}{*}{ Carbonato } & 10 & 348,7 & 395,9 & 614,0 \\
\hline & 20 & 314,9 & 358,6 & 552,0 \\
\hline & 40 & 332,1 & 375,0 & 556,0 \\
\hline & 80 & 373,9 & 416,0 & 608,0 \\
\hline & 150 & 405,5 & 446,0 & 627,0 \\
\hline Mezcla baja & & 335,3 & 381,5 & 561,0 \\
\hline Mezcla media & & 354,0 & 406,0 & 603,0 \\
\hline Mezcla alta & & 433,5 & 479,9 & 655,0 \\
\hline
\end{tabular}

Rev. Metal. Madrid 37 (2001)

http://revistademetalurgia.revistas.csic.es 
obtenidas con el par bola-bornita, con los mismos iones en solución. Se obtiene que 150 ppm de ion cloruro generan, nuevamente, la más alta diferencia de potencial, siendo éste de $411,7 \mathrm{mV}$; el ion sulfato, con $5 \mathrm{ppm}$, genera un potencial del par de $377 \mathrm{mV}$; el ion nitrato, con $50 \mathrm{ppm}$, produce una diferencia de $360 \mathrm{mV}$; el ion carbonato genera la más alta diferencia de potencial a los $150 \mathrm{ppm}$, siendo éste de $446 \mathrm{mV}$ y, finalmente, la mezcla alta de iones genera un potencial de $479,9 \mathrm{mV}$.

Por su parte el par bola-calcopirita presenta las mayores diferencias de potencial, siendo éstos del orden de 550 a $650 \mathrm{mV}$. Las diferencias de potenciales más altos se alcanzan con 150 ppm de cloruro, siendo de $648 \mathrm{mV}$; con $5 \mathrm{ppm}$ de sulfato se obtienen $567 \mathrm{mV}$; con $50 \mathrm{ppm}$ de nitrato, $570 \mathrm{mV}$; con 150 ppm de carbonato se obtienen $627 \mathrm{mV}$ y con la mezcla alta de iones se obtiene un potencial de $655 \mathrm{mV}$.

Es destacable la acción del ion cloruro y el ion carbonato para todos los pares bola-mineral utilizados, presentando las más altas diferencias de potencial. En cambio los iones sulfato y nitrato, mantuvieron siempre menores diferencias de potencial que los obtenidos con los otros iones. Esto se puede deber, principalmente, a que las concentraciones de cloruro y carbonato adicionadas a las soluciones son similares y elevadas y, en cambio, las concentraciones de sulfato y nitrato son bastante inferiores, siendo su efecto también menor. Cabe recordar que las cantidades de cada ion se corresponden con las proporciones del agua industrial analizada.

Cuando existen mezclas de iones, aumenta la fuerza electromotriz (diferencia de potencial) del par bola-mineral. Así, al tener una solución con una mezcla baja de iones, también se obtienen valores bajos de potencial. En cambio, al tener una solución con una mezcla alta de iones, los potenciales obtenidos suben, también, drásticamente.

Además es importante el efecto que cada mineral aportaría a la corrosión del medio de molienda por efecto galvánico. Esto queda de manifiesto al comparar las diferencias de potencial entre los distintos pares. Se puede apreciar que el par bola-calcopirita es el que presenta los mayores valores de fuerza electromotriz, con una diferencia de potencial que siempre está entre los 550 y $650 \mathrm{mV}$, independientemente del ion en solución y muy por encima de los 300 a $400 \mathrm{mV}$ del par bola-bornita y de los 350 a $450 \mathrm{mV}$ del par bola-enargita.

Las mayores diferencias de potencial se obtienen cuando el ion cloruro está presente con una concentración de 150 ppm. De igual forma, el ion sulfato genera potenciales altos al estar presente en una concentración de $5 \mathrm{ppm}$, el ion nitrato con 50 ppm y el ion carbonato con 150 ppm. Estas concentraciones se repiten independiente del par bola-mineral utilizado.

\subsection{Densidades de corriente y potenciales de corrosión para el sistema $\mathrm{Fe} / \mathrm{Fe}^{+2}-\mathrm{O}_{2} / \mathrm{H}_{2} \mathrm{O}$}

En los resultados mostrados anteriormente se determinaron las concentraciones más agresivas de iones en términos de condición termodinámica a través de las diferencias de potencial bola-mineral.

A continuación se presentan las densidades de corriente y potenciales de corrosión obtenidos al utilizar esas concentraciones de iones. Se prepararon 5 soluciones con las concentraciones que se indican:

- Solución 1: 150 ppm de Cloruro

- Solución 2: 5 ppm de Sulfato

- Solución 3: 50 ppm de Nitrato

- Solución 4: 150 ppm de Carbonato

- Solución 5: Mezcla de los iones y concentraciones anteriores

Se obtuvieron los diagramas de Evans para el sistema bola-oxígeno con cada una de las soluciones anteriores. Los diagramas de Evans representan las curvas anódica y catódica con intersección en un único punto (Fig. 3). Dicho punto indica la densidad de corriente y el potencial de corrosión para el sistema en estudio, en este caso oxidación del hierro y reducción del oxígeno. Los diagramas experimentales para cada solución en estudio se pueden apreciar en las figuras 4 a 8 y los valores de las densidades de corriente y potenciales de corrosión obtenidos se han resumido en la tabla V.

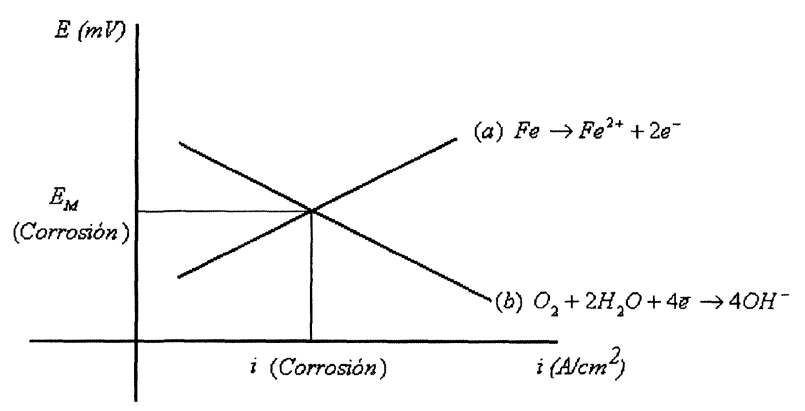

Figura 3. Un ejemplo de un Diagrama de Evans.

Figure 3. A example of a Evans Diagram. 


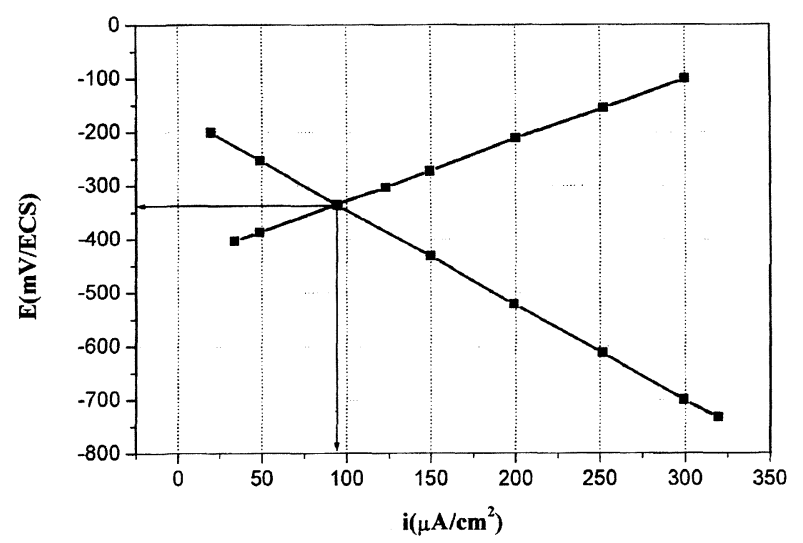

Figura 4. Diagrama de Evans experimental para el sistema bola-cloruro.

Figure 4. Experimental Evans Diagram for the ball-cloride system.

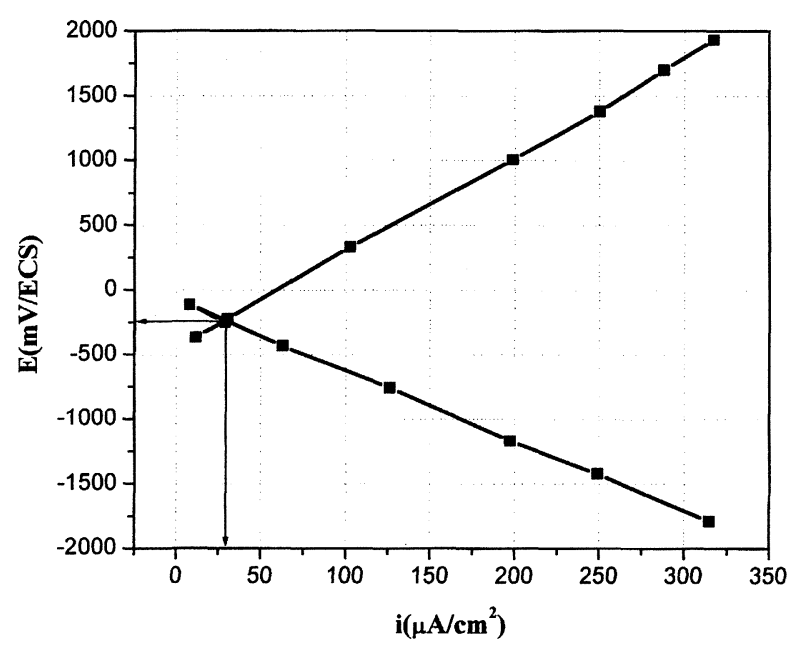

Figura 5. Diagrama de Evans experimental para el sistema bola-sulfato.

Figure 5. Experimental Evans Diagram for the ball-sulfate system.

Tabla V. Densidades de corriente y potenciales de corrosión medidos con diferentes concentraciones de iones

Table V. corrosion current densities and potentials variation with different ionic concentrations.

\begin{tabular}{ccc}
\hline Solución & $\begin{array}{c}\text { Densidad de corriente } \\
\text { de corrosión } \\
\mu \mathrm{A} / \mathrm{cm}^{2}\end{array}$ & $\begin{array}{c}\text { Potencial de } \\
\text { corrosión } \\
\mathrm{mV} / \mathrm{ECS}\end{array}$ \\
\hline 1 & 95,60 & $-337,10$ \\
2 & 28,83 & $-249,90$ \\
3 & 66,59 & $-311,30$ \\
4 & 35,10 & $-144,50$ \\
5 & 150,00 & $-347,00$ \\
\hline
\end{tabular}

8

(c) Consejo Superior de Investigaciones Científicas Licencia Creative Commons 3.0 España (by-nc)

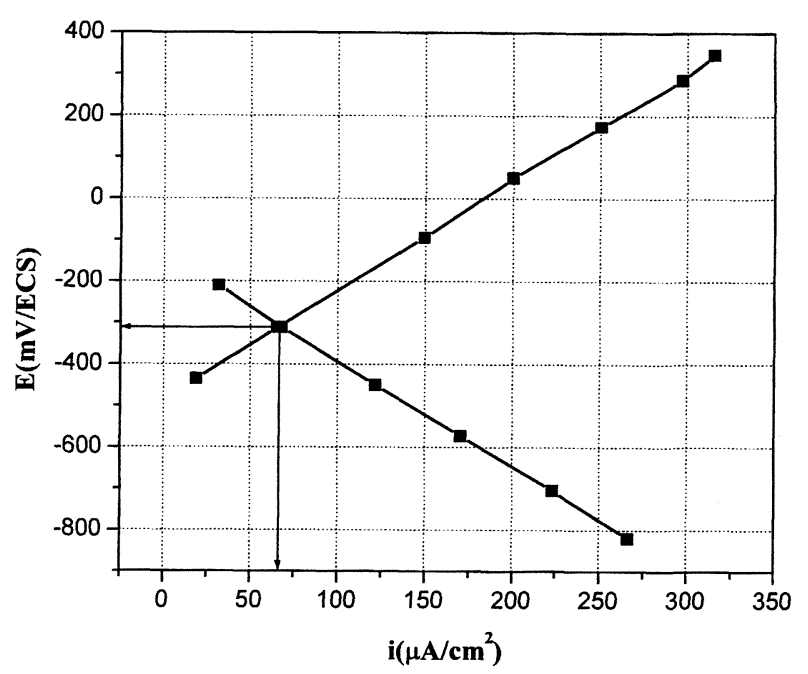

Figura 6. Diagrama de Evans experimental para el sistema bola-nitrato.

Figure 6. Experimental Evans Diagram for the ball-nitrate system.

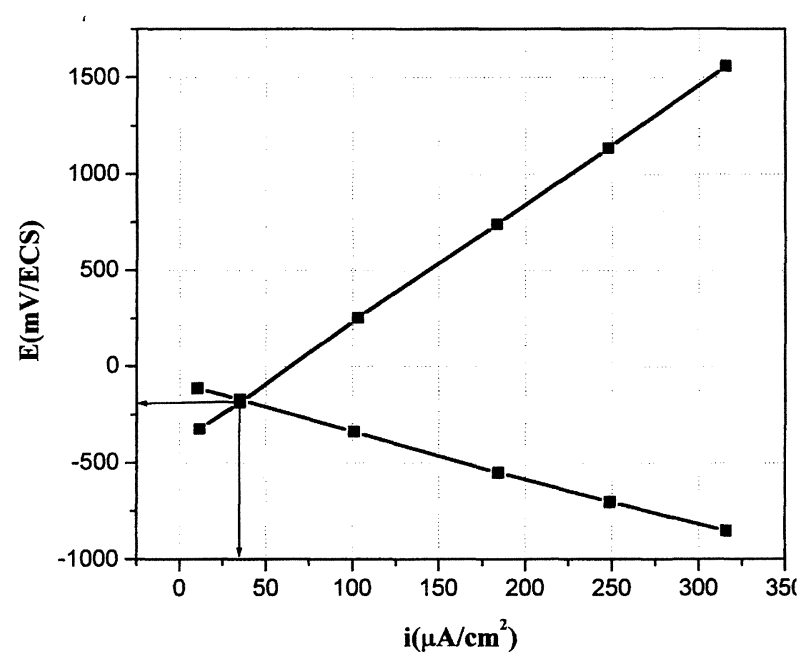

Figura 7. Diagrama de Evans experimental para el sistema bola-carbonato.

Figure 7. Experimental Evans Diagram for the ballcarbonate system.

El ion cloruro es el que generó una mayor cinética de corrosión con una densidad de corriente del orden de los $96 \mu \mathrm{A} / \mathrm{cm}^{2}$. El ion sulfato, en cambio, presentó una cinética menor, del orden de los $29 \mu \mathrm{A} / \mathrm{cm}^{2}$, el ion nitrato de $67 \mu \mathrm{A} / \mathrm{cm}^{2}$ y el carbonato un valor de $35 \mu \mathrm{A} / \mathrm{cm}^{2}$. Cuando hay una mezcla de los iones antes mencionados se obtuvo una mayor densidad de corriente, del orden de $150 \mu \mathrm{A} / \mathrm{cm}^{2}$ y el potencial de corrosión alcanzado es cercano al valor obtenido cuando se utilizó solo cloruro, lo cual está indicando la predominancia de este ión sobre los otros.

Además, como una forma de confirmar lo encontrado anteriormente se obtuvo un diagrama

Rev. Metal. Madrid 37 (2001) 


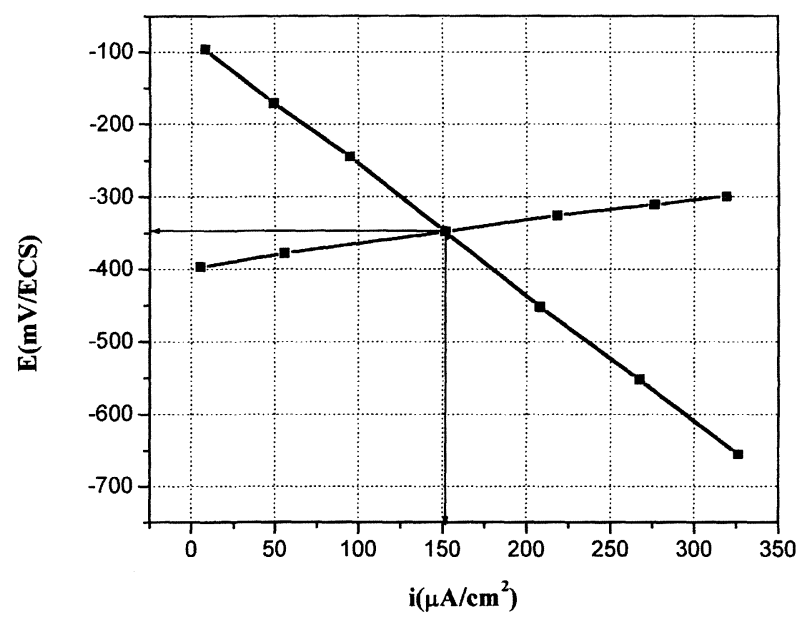

Figura 8. Diagrama de Evans experimental para el sistema bola-mezcla de iones.

Figure 8. Experimental Evans Diagram for the ball-mixture of ions system.

comparativo entre las diferentes rectas anódicas experimentales (Fig. 9). Se observa una clara diferencia entre las pendientes de las rectas experimentales, verificándose que el daño corrosivo se incrementa en el siguiente orden de iones: sulfato $>$ carbonato $>$ nitrato $>$ cloruro, debido a que una menor pendiente genera una mayor velocidad de corrosión. La recta de menor pendiente corresponde a la mezcla de iones, reflejándose esto en una cinética de corrosión mayor provocada por el efecto sinérgico de los iones en cuestión.

\section{CONCLUSIONES}

Para los pares bola-mineral, la calcopirita es la que presentó la mayor diferencia de potencial mixto en

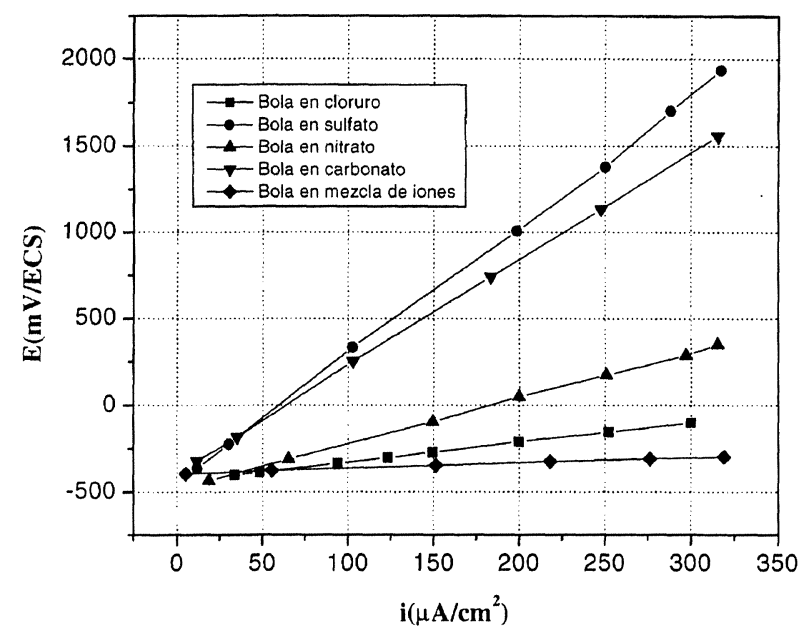

Figura 9. Diagrama comparativo de las rectas anódicas experimentales.

Figure 9. Comparative Diagram of the experimental anodic lines.

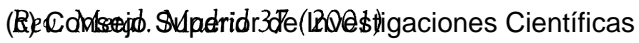

Licencia Creative Commons 3.0 España (by-nc) relación al de la bola, lo que entrega una condición termodinamica más favorable a la corrosión por efecto de par galvánico, seguida por la enargita y finalmente la bornita.

El par bola-mineral alcanza la mayor diferencia en los potenciales mixtos cuando el ion cloruro está presente en una concentración de 150 ppm. De igual forma, el ion carbonato alcanza su mayor efecto corrosivo sobre el par a $150 \mathrm{ppm}$, el ion sulfato a $5 \mathrm{ppm}$ y, finalmente, el nitrato a $50 \mathrm{ppm}$.

En términos de las densidades de corriente de corrosión encontradas, el ion cloruro es el que produciría mayor daño en la molienda húmeda de minerales, luego, el ion nitrato, después el ion carbonato y, por último, el ion sulfato.

De acuerdo a los estudios realizados, la componente de corrosión electroquímica de medios de molienda es importante en presencia de minerales tales como: calcopirita, bornita y enargita; y de iones tales como: cloruros, sulfatos, nitratos y carbonatos. Todo lo cual redunda en un mayor consumo de estos.

\section{AGRADECIMIENTOS}

Los autores expresan su agradecimiento por el patrocinio recibido de la Comisión Nacional de Investigación Científica y Tecnológica a través del proyecto FONDECYT 1950572 y de la Universidad de Santiago de Chile a través del proyecto DICYT 9314MO.

\section{REFERENCIAS}

[1] L. Magne, Tesis de grado, Programa de Magister en Ciencias de la Ing., Mención Metalurgia, Universidad de Concepción (Chile), 1989.

[2] M. Meulendyke, P.J. Moroz y P. Smith, IV Simposium Sobre Molienda, Armco-Chile, Viña del Mar, Chile, 1984, pp. 159-169.

[3] J. ORMEÑo, Trabajo de Titulación, Facultad de Ingeniería, Universidad de Santiago de Chile (Chile), 1993.

[4] R. Pérez, J. MoOre y K. Smith, Annual Meeting SME/AIME, Los Angeles, California, EE.UU., 1984.

[5] P.J. Moroz, V Simposium Sobre Molienda Armco-Chile, Viña del Mar, Chile, 1987, pp. 243-253.

[6] J. Iwasaki, K. Natarajan et Al., Int. J. Miner. Process 22 (1988).

[7] K.A. Natarajan, S.C. Riemer y J. Iwasaki, Int. J. Miner. Process 13 (1984) 73-81.

[8] K.A. Natarajan y J. Iwasaki, Int. J. Miner. Process 13 (1984) 53-71.

[9] A. ISAACSO, Bureau of Mines 9244, 1989.

[10] R. Rebolledo, Trabajo de Titulación, Facultad de Ingeniería Universidad de Santiago de Chile (Chile), 1989. 